\title{
Optical anisotropy of $\mathrm{SrTiO}_{3}(110)$ for different surface terminations
}

\author{
Karsten Fleischer*., Sangsoo Kim, Brian Walls, Kuanysh Zhussupbekov, Igor V. Shvets \\ School of Physics, Trinity College Dublin, The University of Dublin, Dublin 2, Ireland
}

Received XXXX, revised XXXX, accepted XXXX

Published online XXXX

Key words: strontium titanate, STO, RAS, reflectance anisotropy, (110) surface

* Corresponding author: e-mail fleisck@tcd.ie, Phone: +353-1-896-3649

In this work we investigate the $\mathrm{SrTiO}_{3}(110)$ surface by reflectance anisotropy spectroscopy (RAS) at varying preparation steps including $e x$-situ wet etching, high temperature oxygen annealing, as well as in-situ vacuum annealing. Different surface terminations show distinctly different RAS spectra which are correlated to an altered surface stoichiometry measured by X-ray photoelectron spectroscopy (XPS) and an altered valence band structure measured by UV-photoelectron spectroscopy (UPS) in cases of conductive samples.

We link the changes in the observed RAS spectra to various surface reconstructions, with particular focus of the signature of $(3 \times 1)$ and $(1 \times 4)$ reconstructed surfaces, and a metallic surface state observed in vacuum annealed $\mathrm{SrTiO}_{3}$.

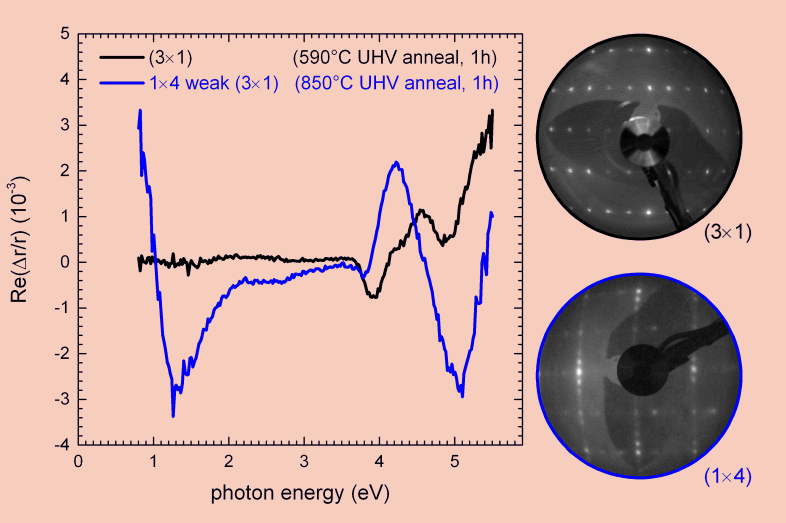

Reflectance anisotropy spectra and LEED images of the $\mathrm{SrTiO}_{3}(110)-(3 \times 1)$ and $(1 \times 4)$ surface

Copyright line will be provided by the publisher

1 Introduction Strontium titanate $\left(\mathrm{SrTiO}_{3}\right)$ is an oxide of perovskite crystal structure with increasing importance as a substrate for heteroepitaxy and superlattices of multiferroic, perovskite oxides [1]. One particular interesting aspect is the formation of a quasi-two-dimensional electron gas (Q2DEG) at the interface of insulating $\mathrm{SrTiO}_{3}$ and $\mathrm{LaAlO}_{3}$ [2]. This hetero interface has been widely investigated in recent years and has shown to have even superconducting properties [3-5]. While most of these studies have been performed on (001) surfaces the formation of this Q2DEG has also been seen on (110) surfaces [6, 7]. In all cases the treatment of the $\mathrm{SrTiO}_{3}$ substrate is a critical step and can influence the interface structure in any hetero-epitaxial system.

For these reasons we started investigating the optical anisotropic response of the $\mathrm{SrTiO}_{3}(110)$ surface by reflectance anisotropy spectroscopy (RAS). RAS is a versa- tile tool, sensitive to surface and interface structures, providing the underlying bulk material is optically isotropic within the plane of incidence [8]. As such RAS has the potential to measure the formation of the Q2DEG in-situ during the heteroepitaxy, as both $\mathrm{SrTiO}_{3}$ and $\mathrm{LaAlO}_{3}$ are wide bandgap materials and the response from the Q2DEG is expected to occur in the infrared spectral region [9]. IR-ellipsometry has already confirmed that the Q2DEG $\mathrm{LaAlO}_{3} / \mathrm{SrTiO}_{3}(110)$ shows inherent anisotropies in terms of the electron mobilities along the [001] and [1 $\overline{1} 0]$ direction [7].

As a first step towards such an experiment, here we investigate the RAS spectra of the $\mathrm{SrTiO}_{3}(110)$ surface prepared by different methods, including ex-situ chemical cleaning, annealing in oxygen atmosphere and in-situ vacuum annealing. We will show that differently terminated $\mathrm{SrTiO}_{3}(110)$ surfaces have distinctly different RAS 
spectra, dominated by surface modified bulk states above the $\mathrm{SrTiO}_{3}$ bandgap, and in case of a vacuum annealed $\mathrm{SrTiO}_{3}(110)-(1 \times 4)$ reconstructed surface a strong infrared response originating from a metallic surface state.

2 Experimental details Undoped $\mathrm{SrTiO}_{3}(110)$ single crystal substrates (MTI Corp.) were used throughout this study. All samples had no nominal offcut, minimising step contributions, and were single sided polished, eliminating any contributions of back surface reflections in RAS and ellipsometric measurements. Preparation conditions were varied to investigate different surface terminations. These include ultrahigh vacuum (UHV) annealing of as-received samples and samples pre-annealed at $950^{\circ} \mathrm{C}$ in air. In a third set additional chemical cleaning in water $\left(80^{\circ} \mathrm{C}\right), \mathrm{HCl}(37 \%): \mathrm{HNO}_{3}(67 \%) 3: 1$ mixed etchant [10], followed by pre-annealing at $950^{\circ} \mathrm{C}$ in air and subsequent UHV annealing steps was investigated.

RAS spectra have been recorded at each preparation step with two in-house built system following the Aspnes design [11]. One system with a broad spectral range of $0.35-5.5 \mathrm{eV}$ was used primarily for studying ex-situ surface preparation. A second more compact system was used for in-situ measurements in the spectral range of 0.8$5.2 \mathrm{eV}$. Details on the two spectrometers are found in [12] and [13] respectively. Residual instrumental anisotropies from polariser nonidealities and strain related signals from UHV windows have been numerical removed by subtracting averaged zeroline signals from isotropic $\mathrm{MgO}(001)$ and $\mathrm{Si}(111)$ reference surfaces. There is a remaining uncertainty in the zero line of $0.2 \times 10^{-3}$ due to sample alignment reproducibility and this error is indicated as error bar on the zero line in all figures. The instruments energy resolution is $0.02 \mathrm{eV}$, estimated by the measured width of sharp xenon emission lines in the lamp's spectrum. In terms of RAS sign and sample orientation, we define $\Delta r / r$ as $2\left(r_{1 \overline{1} 0}-r_{001}\right) /\left(r_{1 \overline{1} 0}+r_{001}\right)$.

Low energy electron diffraction (LEED) patterns, Xray photo-electron and UV-photo-electron spectra (XPS, UPS) have been recorded in an Omicron MultiProbe-XPS system using a monochromated $\mathrm{Al}-\mathrm{K} \alpha$ and a $\mathrm{He}(\mathrm{I}) \mathrm{UV}-$ lamp. All $\mathrm{SrTiO}_{3}(110)$ samples were insulating prior to UHV annealing and XPS measurements were performed using a low energy electron flood gun (CN10) for charge compensation. In these cases LEED and UPS measurements are missing due to sample charging issues.

In-situ sample annealing was performed initially on a resistive heat stage, using flat Ta-plates with a sample held in place by Ta-strips spot welded onto the plate. As the sample surface temperature was limited to $850^{\circ} \mathrm{C}$ a second sample holder was constructed with a cut-out for the $\mathrm{SrTiO}_{3}$ sample, which was than clamped into the holder by a Ta circlip. This design allowed for a better thermal contact of the sample to the sample plate and more efficient radiative heat transfer from the heater to the sample itself. UHV annealing for $1 \mathrm{~h}$ in the second case resulted in similar surface reconstructions as previously seen for short flash anneal cycles of up to $1400^{\circ} \mathrm{C}$ [14-16]. To prepare for future scanning tunneling microscopy work (STM) and investigate if the heating ramp rate influences results, a final set of UHV-annealing steps of a chemically etched sample was carried out in a Createc STM chamber with an e-beam heater. Specifically the onset of the formation of bulk oxygen vacancies and their influence on the RAS spectra was investigated by UHV annealing in steps of $100 \mathrm{~K}$ between 500 and $1100^{\circ} \mathrm{C}$. In a final set of measurements the samples went through three additional sputter/anneal cycles using Ar-ions $\left(0.6 \mathrm{kV}, 1 \times 10^{-5} \mathrm{mbar}\right.$ for $20 \mathrm{~min}$ and $500^{\circ} \mathrm{C}$ anneal for $20 \mathrm{~min}$ ) prior extended UHV annealing in order to compare our sample surfaces with previous reported surface preparations [15].

Table 1 summarises the various treatments, lists the reconstruction observed based on their LEED pattern, as well as gives each preparation a unique naming identifier to be used in the figures showing the corresponding RAS spectra.

3 Results The aim of this study was to investigate the surface optical anisotropy of well defined $\mathrm{SrTiO}_{3}(110)$ surfaces. One difficulty is that the (110) surface is less studied than the (001) surfaces and that a wide range of surface reconstruction has been observed depending on preparation conditions $[14,15,17]$. Generally the observed surface reconstructions can be classified into two categories; The first is the relatively well understood $(n \times 1)$ family $(2 \leq n \leq 6)$ found under oxidising conditions $[15,18]$. These surfaces are believed to be titanium rich, originating from a long range order of $\mathrm{TiO}_{2}$ corner sharing tetrahedra on top of subsurface $\mathrm{TiO}_{2}$ octahedra with various row spacings [18].

Other surfaces formed under other conditions including high temperature UHV annealing, sputter annealing cycles or $\mathrm{Ti}$ and $\mathrm{Sr}$ adsorption include $(2 \times 5),(3 \times 4)$, $(4 \times 4),(4 \times 7),(6 \times 4), c(2 \times 6)$ reconstructions $[14,15,17$, 19]. Their individual nature however is much less understood. Due to the restriction of our experimental setup, we will only discuss surface reconstructions found by insitu UHV annealing from 500 to $1100^{\circ} \mathrm{C}$ with differing substrate pre-cleaning procedures.

3.1 Direct surface de-oxidation As a first step the direct surface decontamination by UHV annealing was investigated by XPS and RAS (see Fig. 1). As-received samples already show a distinct RAS spectra with a characteristic minima and maxima $3.85 \mathrm{eV}$ and $4.45 \mathrm{eV}$. As any significant RAS signal occurs above the band gap of $\mathrm{SrTiO}_{3}$, the anisotropy of the as-received sample is interpreted as the result of the surface induced anisotropy of any cubic (110) surface [11]. The intrinsic anisotropy of the (110) surface unit cell, combined with potential electric field gradients, strain gradients, coherent uniaxial strain, or localised surface states can all induce a small optical anisotropy in an otherwise optically isotropic material. A 
Table 1 Overview of the different sample preparations and achieved surface terminations (from LEED). The ID will be used in the following to refer to individual samples, the color of the ID corresponds to the color of the RAS spectra in Figures 1-5. Vacuum annealing was done in UHV conditions (below $1 \times 10^{-9}$ mbar). Conditions which resulted in a single domain surface have been highlighted.

\begin{tabular}{|c|c|c|c|}
\hline ID & Preparation & LEED & Contaminants \\
\hline \multicolumn{4}{|c|}{ No pretreatment, sample as received from supplier } \\
\hline S1-asreceived & as received & too insulating & $\mathrm{C}, \mathrm{OH}$ \\
\hline S1-vac500 & $500^{\circ} \mathrm{C}$ UHV annealing, $1 \mathrm{~h},<1 \times 10^{-9}$ mbar & $(2 \times 1), c(2 \times 2)$ & $\mathrm{C}$ \\
\hline S1-vac800 & $800^{\circ} \mathrm{C}$ UHV annealing, $1 \mathrm{~h},<1 \times 10^{-9}$ mbar & $c(2 \times 2)$ & $\mathrm{C}$ \\
\hline \multicolumn{4}{|c|}{ Sample annealed in air at $950^{\circ} \mathrm{C}$ for $2 \mathrm{~h}$ prior loading into the UHV chamber } \\
\hline S2-air950 & as loaded & too insulating & residual C \\
\hline S2-air950-vac500 & $500^{\circ} \mathrm{C} \mathrm{UHV}$ annealing, $1 \mathrm{~h}$ & $(3 \times 1)$ & \\
\hline S2-air950-vac750 & $750^{\circ} \mathrm{C}$ UHV annealing, $1 \mathrm{~h},<1 \times 10^{-9} \mathrm{mbar}$ & $(3 \times 1),(1 \times 4)$ & \\
\hline \multicolumn{4}{|c|}{ Sample etched at $75^{\circ} \mathrm{C}$ in $\mathrm{HCl}(37 \%): \mathrm{HNO}_{3}(67 \%)$ 3:1 mixed acid and annealed in air at $950^{\circ} \mathrm{C}$ for $2 \mathrm{~h}$} \\
\hline S3-chem & as loaded & too insulating & residual C \\
\hline S3-chem-vac750 & $750^{\circ} \mathrm{C}$ UHV annealing, $1 \mathrm{~h},<1 \times 10^{-9} \mathrm{mbar}$ & $(1 \times 4),(3 \times 1)$ & \\
\hline S3-chem-vac850 & $850^{\circ} \mathrm{C}$ UHV annealing, $1 \mathrm{~h},<1 \times 10^{-9}$ mbar & $(1 \times 4),(3 \times 1)$ & \\
\hline S4-chem & as loaded, repeat of S3-chem on new sample & too insulating & \\
\hline S4-chem-vac500 & $500^{\circ} \mathrm{C}$ UHV annealing, $1 \mathrm{~h},<1 \times 10^{-9}$ mbar & $(1 \times 4),(3 \times 1)$ & \\
\hline S4-chem-Ar-vac1100 & $3 \times$ Ar sputter/anneal cycle, final UHV anneal at $1100^{\circ} \mathrm{C}, 1 \mathrm{~h}$ & $(\mathbf{1} \times \mathbf{4})$ & \\
\hline \multicolumn{4}{|c|}{ specific additional modifications to previous samples } \\
\hline S5-chem & Repeated chemical etch and air anneal of sample S3-chem-vac850 & too insulating & \\
\hline S5-chem-vac500 & $500^{\circ} \mathrm{C}$ UHV annealing, $1 \mathrm{~h},<1 \times 10^{-9} \mathrm{mbar}$ & $(1 \times 4),(3 \times 1)$ & \\
\hline S5-chem-vac1000 & $1000^{\circ} \mathrm{C}$ UHV annealing, $1 \mathrm{~h},<1 \times 10^{-9} \mathrm{mbar}$ & $(1 \times 4),(3 \times 1)$ & \\
\hline S5-exposed & exposure of sample S5-chem-vac900 to air & disordered & \\
\hline S5-exposed-vac500 & $500^{\circ} \mathrm{C}$ UHV annealing, $1 \mathrm{~h},<1 \times 10^{-9} \mathrm{mbar}$ & $(1 \times 4),(3 \times 1)$ & \\
\hline
\end{tabular}


Figure 1 (a) RAS spectra of an as-received $\mathrm{SrTiO}_{3}(110)$ sample (....) and after subsequent UHV annealing for $1 \mathrm{~h}$ at

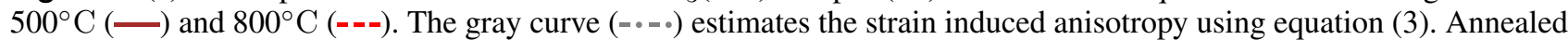
samples were conductive enough to take LEED images showing a weakly ordered $(2 \times 1) / \mathrm{c}(2 \times 2)$ pattern. (b) XPS data for the as-received and $500^{\circ} \mathrm{C}$ UHV annealed sample showing a removal of hydroxide groups.

strain related RAS signal, typically creates structures in the vicinity of a materials bulk critical points. The relationship between the bulk dielectric function and RAS structures have been extensively discussed in terms of bulk strain, but also surface and step induced strain in silicon [20,21]. For the Si(110) surface the resulting spectra are found to be combinations of strain and other effects, and also hydrogen can play a crucial role $[11,22,20]$. For the $\mathrm{SrTiO}_{3}$ 
surfaces investigated here, no detailed calculation on the optical properties of various surfaces, nor detailed adsorption studies exist. Hence we can only discuss spectra with qualitative models, in particular the possible effect of surface induced strain. Within a crude strain model, one can simulate the spectral shape of such strain induced structures via the three layer model [23] by equating the surface dielectric anisotropy with the first derivative of the bulk dielectric function multiplied by an estimate of the thickness of the strained region

$$
d\left(\varepsilon_{x}-\varepsilon_{y}\right)=d \Delta \varepsilon_{s} \approx d \frac{\mathrm{d} \varepsilon_{b}}{\mathrm{~d} E}
$$

With the three layer model for RAS:

$$
\frac{\Delta r}{r}=\frac{4 \pi i d}{\lambda} \frac{\varepsilon_{x}-\varepsilon_{y}}{\varepsilon_{b}-1}
$$

the strain contribution in the measured RAS can then be simulated using the expression for $\operatorname{Re}(\Delta r / r)$ from [8]:

$$
\operatorname{Re}\left(\frac{\Delta r}{r}\right)=\frac{4 \pi d}{\lambda}\left(\frac{\left(\varepsilon_{1}-1\right) \frac{\mathrm{d} \varepsilon_{2}}{\mathrm{~d} E}-\varepsilon_{2} \frac{\mathrm{d} \varepsilon_{1}}{\mathrm{~d} E}}{\left(\varepsilon_{1}-1\right)^{2}+\varepsilon_{2}^{2}}\right)
$$

The bulk dielectric function of the $\mathrm{SrTiO}_{3}\left(\varepsilon_{b}=\varepsilon_{1}+i \varepsilon_{2}\right)$ was measured by ellipsometry (Sopra GESP5) on the same crystal as RAS measurements have been performed. The result of this simulation is shown in Fig. 2, using a value for $d$ of $12 \mathrm{~nm}$. The latter was chosen to reproduce the amplitude of the minimum structure at $3.9 \mathrm{eV}$. The simple strain models shows some similarities with the measured structure, particularly just above the direct band gap of $\mathrm{SrTiO}_{3}$ at $3.7 \mathrm{eV}$. However it fails to describe the structures at other transitions, such as the indirect gap at $3.25 \mathrm{eV}$ and higher energy transitions $\left(A_{1}, A_{2}\right)$. There are also considerable variations in the reported dielectric function of $\mathrm{SrTiO}_{3}$ due to the ternary nature and variations in oxygen stoichiometry in bulk crystals [24]. Here we use the terminology to label transitions following the work of van Benthem et al. [24], while the energetic positions have been determined by analysing the second derivative of ellipsometric measurements of the bulk crystals used in this work. They are found to be broadly consistent with those in [24], giving an indirect gap $E_{i}=3.29 \mathrm{eV}$; direct gap $E_{d}=3.70 \mathrm{eV}$; transition $A_{1}=4.21 \mathrm{eV}$; and transition $A_{2}=4.76 \mathrm{eV}$. These energies are included as vertical lines to guide the eye in all shown RA spectra.

The insulating nature of the as-received sample does not allow for a measurement of any LEED pattern to analyse any residual coherent surface symmetry. UHV annealing at $500^{\circ} \mathrm{C}$ creates enough bulk oxygen vacancies to prevent sample charging and a weak LEED pattern is observed. However the $(2 \times 1)$ LEED pattern is not well defined with a superimposed $\mathrm{c}(2 \times 2)$ structure. XPS measurements reveal significant remaining carbon contamination, with the only substantial change being the removal of a second component in the $\mathrm{O} 1 \mathrm{~s}$ core level typically associated with the presence of hydroxide or carbonate groups in the surface region [25,26]. The RAS spectra however clearly change, with a change of the prominent $4.45 \mathrm{eV}$ maxima. Further annealing of this surface to $800^{\circ} \mathrm{C}$ for an hour did not lead to a significantly better ordered surface, although dominated by a weak $\mathrm{c}(2 \times 2)$. The RAS shows a further reduction in intensity around $4.5 \mathrm{eV}$ and a broad minimum within the band gap, indicating an increase in point defects within the surface region. To our knowledge, a c $(2 \times 2)$ structure has not been previously investigated, but the surface was not well ordered, and a significant carbon contamination remained. Hence further substrate pre-cleaning steps have been investigated.

\section{2 ex-situ surface pre-cleaning}

3.2.1 $950^{\circ} \mathrm{C}$ air annealing Previous reports on air or oxygen annealed $\mathrm{SrTiO}_{3}(110)$ surfaces, lead to the family of $(n \times 1)$ reconstructions. Temperatures in the range of $900-1000^{\circ} \mathrm{C}$ and annealing times of $30 \mathrm{~min}$ to several hours have been used $[14,15]$. For this work we used ambient air annealing at $950^{\circ} \mathrm{C}$ for $2 \mathrm{~h}$. After this procedure the substrates remain fully insulating and LEED patterns (see Fig. 2) were only seen after further UHV annealing at $500^{\circ} \mathrm{C}$. There are, however, no significant changes in the RAS spectra before and after this gentle UHV annealing, indicating the only change in the sample is a change in bulk conductivity, but not the surface termination. We can therefore conclude that $950^{\circ} \mathrm{C}$ air annealing leads to a single domain $(3 \times 1)$ surface with a RAS spectra characterised by a sharp minima at $3.90 \mathrm{eV}$ and strong positive signal in the UV above $4.5 \mathrm{eV}$ (Fig. 2a, - - ). The RAS spectra of $500^{\circ} \mathrm{C}$ UHV annealed samples without any pre-treatment (Fig. 1a, -) are very similar to those, consistent with the analysis of Enterkin et al. [18] that the $(\mathrm{n} \times 1)$ reconstructions $(\mathrm{n}=1$ $6)$ are all related. Indeed there is very little difference in the RAS of the sharp $(3 \times 1)$ and the mixed $(2 \times 1) / c(2 \times 2)$ surface (S2-air950-vac500, S1-vac500). Only the level around the $A_{1}$ transition differs. Both spectra show the characteristic minima at $3.9 \mathrm{eV}$, just above the direct gap $E_{d}$. As previous STM work and DFT studies for the $(3 \times 1)$ show a distinct row structure $[15,18]$, and the RAS fine structure within the rising slope in the UV is reproduced by the simple strain model, the RAS of these two surfaces is likely to be dominated by the anisotropic strain of the row reconstruction to the underlying $\mathrm{SrTiO}_{3}$ bulk.

In the case of the air annealed samples, further annealing in UHV at higher temperatures lead to a blurring of the $(3 \times 1)$ reconstruction and appearance of weak $(1 \times 4)$ spots suggesting a coexistence of a $(3 \times 1)$ and $(1 \times 4)$ termination. This termination has been previously referred to as "(3×4)" LEED pattern [14] and it was already suggested by STM studies of Russellet al. [15] that these related to two different terminations coexisting. In terms of the RAS spectra the appearance of the $(1 \times 4)$ spots is accompanied by an intense maxima minima structure at the $A_{1}$ and $A_{2}$ transitions. In the following we will show that modifications of our heating stage, combined with chemical pre- 

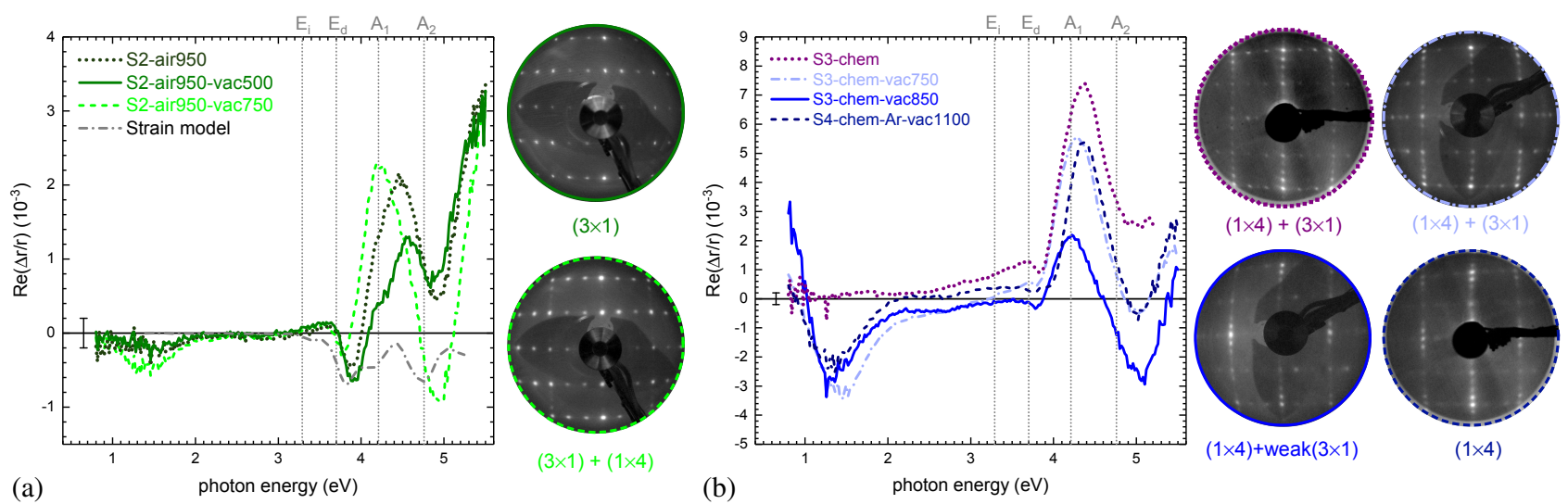

Figure 2 (a) RAS spectra of a $\operatorname{SrTiO}_{3}(110)$ sample (....) after air annealing and subsequent UHV annealing for $1 \mathrm{~h}$ at $500^{\circ} \mathrm{C}$

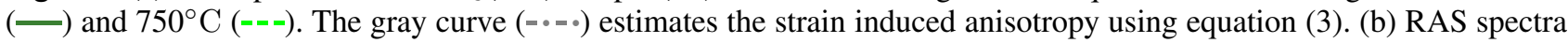
of an air annealed sample which was pre-etched by an $\mathrm{HCl}(37 \%): \mathrm{HNO}_{3}(67 \%)$ 3:1 mixed acid (...) and further stepwise

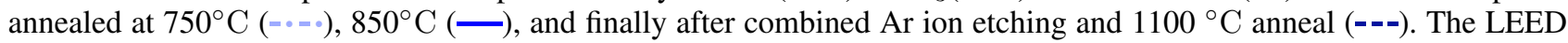
images were taken for the same surfaces at $60 \mathrm{eV}$.

treatment allows for the preparation of a almost single domain $(1 \times 4)$ reconstructions and we will discuss its details in the following section.

3.2.2 Chemical etching plus air anneal For (001) substrates Kareev et al. reported that a chemical precleaning method employed prior air annealing can result in a smooth, well ordered $\mathrm{TiO}_{2}$ terminated surface [10]. We therefore investigated the effect of this method on the RAS spectra of $\mathrm{SrTiO}_{3}(110)$. Substrates are kept for $20 \mathrm{~min}$ in a deionised water bath at $80^{\circ} \mathrm{C}$ followed by a 10 min etching in $\mathrm{HCl}(37 \%): \mathrm{HNO}_{3}(67 \%) 3: 1$ mixed acid, deionised water rinse, and final $\mathrm{N}_{2}$ blow drying. All chemical cleaning steps were done in ambient light. Following this procedure the same $2 \mathrm{~h}$ ambient air anneal was performed as for the previous samples. RAS spectra after the final air annealing show clear differences to those without chemical pre-treatment. The spectra is dominated by a large maximum around $4.4 \mathrm{eV}$ in the vicinity of the $A_{1}$ transitions. The minimum structure, dominating the $(3 \times 1)$ and $(2 \times 1)$ RAS is still present, though less dominant due to the much larger maximum at $4.4 \mathrm{eV}$ (please note the significant change in scale between Fig. $2 \mathrm{a}$ and $\mathrm{b}$ ).

In contrast to previous samples, those pre-etched in the $\mathrm{HCl}(37 \%): \mathrm{HNO}_{3}(67 \%) 3: 1$ mixed acid already show a mixed $(3 \times 1) /(1 \times 4)$ terminated surface after the initial gentle $500^{\circ} \mathrm{C}$ UHV anneal to increase the sample conductivity. RAS spectra before and after this $500^{\circ} \mathrm{C}$ anneal, again, only show small changes (see Fig. 3 C,D) likely related to desorption of physisorbed carbon residues (see also XPS data in Fig. 4) rather than alterations of the surface reconstruction. It is therefore likely the mixed $(3 \times 1) /(1 \times 4)$ reconstruction is already present prior any UHV annealing.

Upon UHV annealing above $600-700^{\circ} \mathrm{C}$ the relative intensity of the $(3 \times 1)$ spots gets weaker compared to the

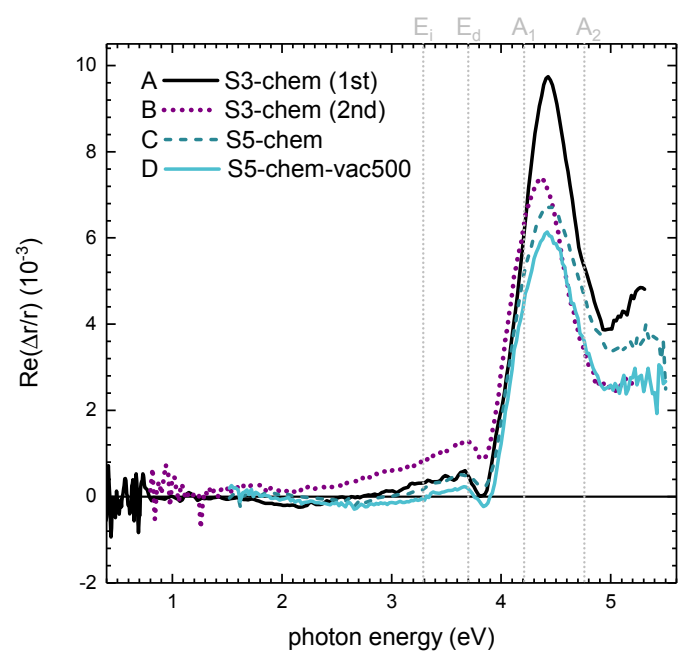

Figure 3 Comparison of RAS spectra after alternating etch/air anneal cycles (A, B), and an etch/air anneal cycle after previous UHV anneal, illustrating the variation in the RAS spectra caused by differences in the resulting $(3 \times 1)$ to $(1 \times 4)$ area ratios and overall surface order. Spectra $D$ is was taken after $500^{\circ} \mathrm{C}$, showing within the variations the same RAS spectra. For this sample LEED measurements have been possible (image shown in Fig. 2, ...) illustrating that etch/air annealed surface have a mixed $(3 \times 1) /(1 \times 4)$ termination.

$(1 \times 4)$ spots, indicating a change in the area ratios of the two reconstruction. The RAS spectra for all these samples are linear combination of the $(3 \times 1)$ spectra and the $(1 \times 4)$ spectra with varying ratios, complicating the analysis significantly.

The $(1 \times 4)$ contribution in all cases has a characteristic strong maxima/minima structure at $4.2 / 5.0 \mathrm{eV}$. With in- 


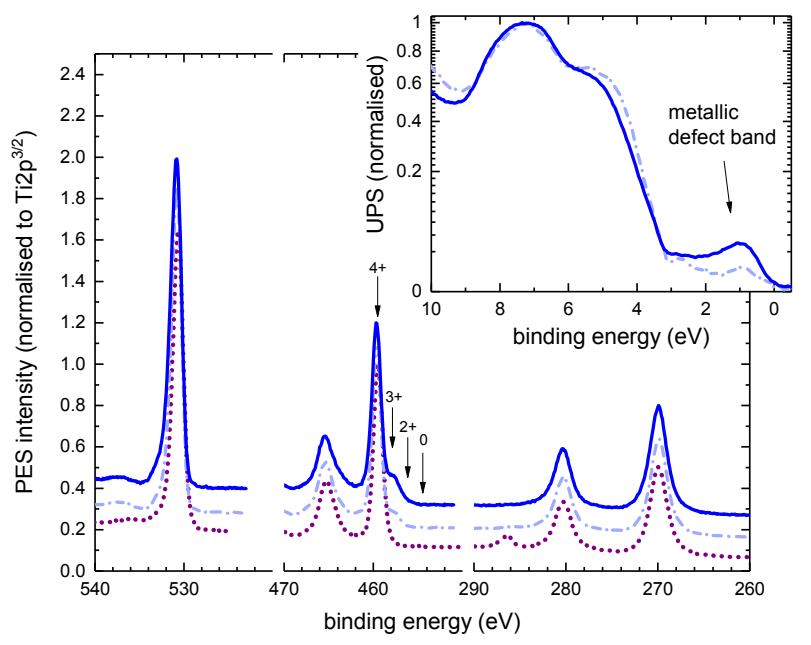

Figure 4 UPS and XPS data for a etched and air annealed sample (...), followed by subsequent UHV annealing at $750^{\circ} \mathrm{C}(-\circ \cdot \circ)$, and $850^{\circ} \mathrm{C}(-)$. The creation of oxygen vacancies leads to an increased bulk conductivity, alterations in the Ti oxidation state with spectral weight for $\mathrm{Ti}^{3+}$ and $\mathrm{Ti}^{2+}$ increasing. This is correlated with the formation of a metallic defect band seen in UPS spectra (inset).

creasing temperature the relative contribution of the $(1 \times 4)$ termination increases, but LEED images also start to blur and an additional minima RAS-structure at $1.4 \mathrm{eV}$ evolves within the $\mathrm{SrTiO}_{3}$ bandgap. The latter is not directly proportional to the $(1 \times 4)$ area but is also seen for the more disordered $c(2 \times 2)$ reconstruction. The below bandgap RAS features will be discussed separately below. The RAS features above the bandgap can now be understood as linear combinations of the $(3 \times 1)$ RAS spectra (S2-air950vac500) and the $(1 \times 4)($ S3-chem-vac850) with varying ratios between $(3 \times 1)$ and $(1 \times 4)$ contributions.

3.3 Below bandgap RAS structures For all high temperature annealed surfaces $\left(T>700^{\circ} \mathrm{C}\right)$ there is a nonnegligible RAS minima developing in the $1.2-1.6 \mathrm{eV}$ region. It initially manifests as a minimum but as it increases amplitude is also accompanied by a sharply rising slope towards the IR and we expect the signal to rise further below $0.8 \mathrm{eV}$. We link the appearance of this infrared signature with the formation of a substantial number of oxygen vacancies within the sample, creating defect states responsible for a) an effective doping of the material with electron accumulation at the surfaces and b) for mid-gap states leading to non vanishing absorption below the band gap, and hence new features in this range. Indeed an absorption feature at $1.3 \mathrm{eV}$ seen in $\mathrm{SrTiO}_{3}$ homoepitaxial thin films, grown in oxygen poor conditions was linked to the formation of Sr-O-O vacancy complexes [27]. Already the bulk conductivity of $\mathrm{SrTiO}_{3}$ itself has been related to oxygen vacancy formation [28]. Recently it was shown that oxygen vacancies in $\mathrm{SrTiO}_{3}$ cluster into vacancy rows along the [001] direction [29]. It is reasonable to assume that the formation of oxygen vacancies is therefore linked to the appearance of the infrared related RAS features, and possibly even be related to the formation of the $(1 \times 4)$ reconstruction itself, in the same way as ordered surface or subsurface oxygen vacancies have been found in other surface reconstructions of transition metal oxides such as $\mathrm{TiO}_{2}$ and $\mathrm{Fe}_{3} \mathrm{O}_{4}[30-32]$.

Figure 4 illustrates that the appearance of the IR structure in the RAS correlates with the appearance of defect states close to the Fermi edge and changes in the Ti $2 p$ core level indicating an increase in $\mathrm{Ti}^{3+}$ and $\mathrm{Ti}^{2+}$ sites, but no metallic $\mathrm{Ti}^{0}$ [28]. For annealing steps up to $850^{\circ} \mathrm{C}$ the creation of the oxygen vacancies does not directly alter the surface termination, except for a slight decrease in the $(3 \times 1)$ spot intensity, but creates defect states and free carriers within the $\mathrm{SrTiO}_{3}$ and increased surface disorder seen by the blurring of LEED spots. The change is significantly more dramatic in the RAS spectra, where the response below $2 \mathrm{eV}$ is substantially altered, even if the defect state in the UPS spectra is still very small. In Fig. 4 a square root scaling of the normalised counts has been used to illustrate the defect band is present for the $750^{\circ} \mathrm{C}$ annealed surface as it is significantly smaller than for further annealing steps. In contrast the RAS amplitude for the same surface at $1.4 \mathrm{eV}$ is already comparable to those of further annealing steps. As RAS measures the ratio of the difference in dielectric response for the [1 $1 \overline{1} 0]$ and [001] direction normalised by the average bulk response it is difficult to directly interpret RAS amplitudes in a quantitative manner, as an increased density of oxygen vacancies, and increased sample conductivity will also alter the bulk reflectivity of the sample. However qualitatively it is clear that the RAS is very sensitive to the formation of the oxygen vacancies.

The presence of a near surface electron gas or metallic surface states, as clearly seen in the UPS data, is also expected to directly lead to a free electron like response in the infrared region of any optical spectra. For RAS measurements, in contrast to simple reflectance measurement, such a free electron response can create an apparent minimum in the difference structure related to $\varepsilon_{x}-\varepsilon_{y}$ even if both $\varepsilon_{x}$ and $\varepsilon_{y}$ are rising monotonically towards the IR [9]. The simulation in Fig. 5 illustrates a possible line shape for such a free electron response using equation (2), and two Drude dielectric functions for $\varepsilon_{x}$ and $\varepsilon_{y}$ with the same plasma frequency but anisotropic scattering rates. The simulation is only indicative, as the spectral range was not wide enough for any form of quantitative fits, however a three times higher mobility for the [1 10$]$ (here $x$ ) compared to the [001] direction was used, consistent with the experimentally found mobility anisotropy in the $\mathrm{SrTiO}_{3} / \mathrm{LaAlO}_{3}(110)$ interface [7]. The observed RAS structure is however most likely a combination of an anisotropy in the polarisability of the near surface oxygen vacancy related mid gap states and the free carrier contribution. 


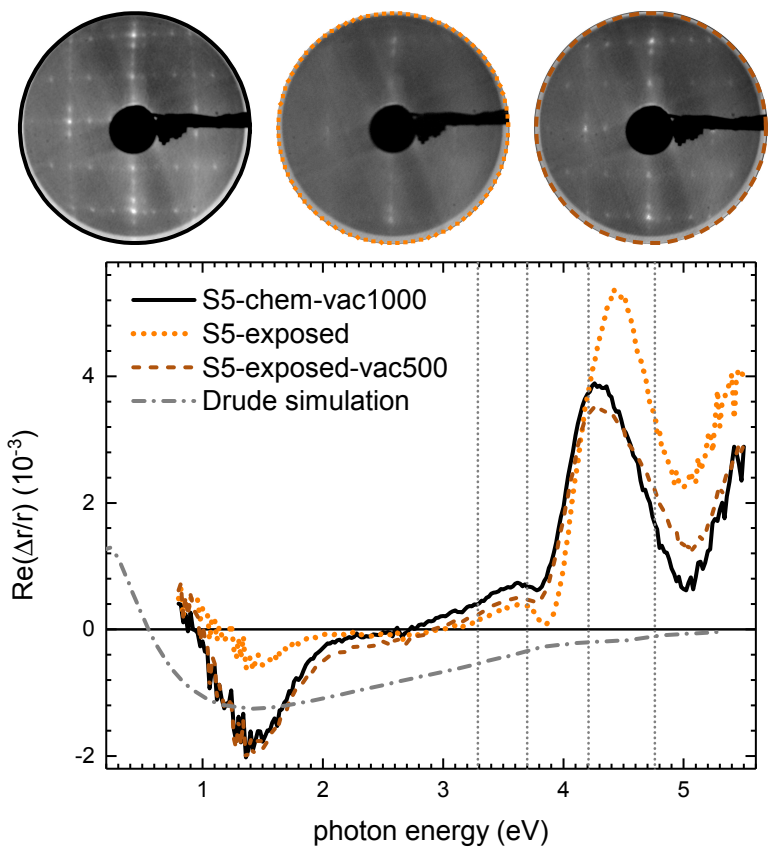

Figure 5 RAS spectra and LEED image of a $1000^{\circ} \mathrm{C} \mathrm{UHV}$ annealed surface prior (-) and after ( $\cdots$ ) air exposure. Subsequent annealing at only $500^{\circ} \mathrm{C}(---)$ fully recovers the original structure. The simulation (-•-•) illustrates a possible free electron contribution to the overall RAS signal.

One aspect to consider is that pre-etched surfaces annealed at 500 and $1000^{\circ} \mathrm{C}$ have very similar LEED patterns, indicating no change in surface termination, but show significantly different infrared RAS responses. This suggests that the infrared RAS signal is more likely to originate from bulk oxygen vacancies rendering the $\mathrm{SrTiO}_{3}$ conductive rather than direct surface alterations. In this case either the surface induced strain gradient leads to an anisotropic signal in the near surface bulk area, or carriers accumulate at the surface in a quasi two-dimensional sheet and we observe an anisotropy in the 2D carrier mobility due to the anisotropic surface termination.

In an attempt to distinguish if the infrared RAS signal originates from the surface layer or a near surface strained bulk, samples have been exposed to air and remeasured. As seen in Fig. 5, air exposure almost fully quenches the RAS minima at $1.4 \mathrm{eV}$. As at the same time the RAS amplitude in the UV even increases, we can conclude that the surface termination induced strain is still present. The disappearance of the IR response then indicates that it originates from the topmost layers and not from an extended near surface bulk-like area as the UV-RAS. Upon gentle annealing at $500^{\circ} \mathrm{C}$ the RAS almost fully recovers. As at similar annealing conditions on samples without any bulk conductivity (see Fig. 3) no infrared signal is created, we do not create new oxygen vacancies, but rather remove ph- ysisorbed species and reveal the underlying original reconstruction, while recovering the electron accumulation layer in the top surface.

4 Conclusions We have illustrated that RAS measurements on $\mathrm{SrTiO}_{3}(110)$ surfaces are sensitive to the surface reconstructions and the formation of a quasi twodimensional electron gas present in UHV annealed samples. Different surface treatments alter the surface order, indicated by different RAS spectra. We were able to prepare two different single domain surface terminations, a $(3 \times 1)$ and $a(4 \times 1)$ reconstruction with distinct RAS spectra (see abstract figure). Samples directly annealed in air result in a single domain $(3 \times 1)$ seen after moderate subsequent UHV annealing, while samples pre-etched in $\mathrm{HCl}: \mathrm{HNO}_{3}$ mixed acid already show a $(3 \times 1)+(1 \times 4)$ mixed surface, likely related to preferential $\mathrm{Sr}$ removal by the etching. High temperature annealing of the latter surface can significantly increase the $(1 \times 4)$ domain. Most surface termination related changes in the RAS spectra occur in the UV above the $\mathrm{SrTiO}_{3}$ bandgap, indicating that surface induced strain is contributing to the RAS signal. In contrast the infrared signal, related to the electron gas and oxygen vacancies, is surface localised, with RAS being even more sensitive to its formation than UPS. This is encouraging for future work on $\mathrm{SrTiO}_{3} / \mathrm{LaAlO}_{3}$ interfaces, as in contrast to electron based techniques it can be employed in-situ during the growth of the heterostructures and can be employed to measure the properties of the interface localised electron gas while it is formed. This is particular critical as typical growth conditions for the $\mathrm{SrTiO}_{3} / \mathrm{LaAlO}_{3}$ interface exceed $750^{\circ} \mathrm{C}$, above the onset temperature for the formation of a significant number of oxygen vacancies $[7,6]$. This is typically mitigated by a sample cool down at higher oxygen pressures (up to 200mbar), which was not possible in our system.

Acknowledgements The authors would like to acknowledge funding from Science Foundation Ireland under grant number 12/IA/1264, and the support of the Government of Republic of Kazakhstan under the Bolashak program. We also would like to thank Christopher Smith for the ellipsometric measurements on our samples. K.F. would like to acknowledge many fruitful discussions and advise from Prof. John F. McGilp in all those past years of trying to understand RAS spectra and beyond.

\section{References}

[1] R. Ramesh and N. A. Spaldin, Nat. Mater. 6, 21 (2007).

[2] A. Ohtomo and H. Hwang, Nature 427, 423 (2004).

[3] N. Reyren, S. Thiel, A. Caviglia, L. F. Kourkoutis, G. Hammerl, C. Richter, C. Schneider, T. Kopp, A. S. Rüetschi, D. Jaccard et al., Science 317, 1196 (2007).

[4] M. Basletic, J. L. Maurice, C. Carrétéro, G. Herranz, O. Copie, M. Bibes, É. Jacquet, K. Bouzehouane, S. Fusil, and A. Barthélémy, Nat. Mater. 7, 621 (2008).

[5] G. Herranz, M. Basletić, M. Bibes, C. Carrétéro, E. Tafra, E. Jacquet, K. Bouzehouane, C. Deranlot, A. Hamzić, J. M. Broto et al., Phys. Rev. Lett. 98, 216803 (2007). 
[6] G. Herranz, F. Sánchez, N. Dix, M. Scigaj, and J. Fontcuberta, Sci. Rep. 2, 758 (2012).

[7] M. Yazdi-Rizi, P. Marsik, B. Mallett, K. Sen, A. Cerreta, A. Dubroka, M. Scigaj, F. Sánchez, G. Herranz, and C. Bernhard, Phys. Rev. B 95, 195107 (2017).

[8] P. Weightman, D. S. Martin, R. J. Cole, and T. Farrell, Rep. Prog. Phys. 68, 1251 (2005).

[9] K. Fleischer, S. Chandola, T. Herrmann, N. Esser, W. Richter, and J.F. McGilp, Phys. Rev. B 74, 195432 (2006).

[10] M. Kareev, S. Prosandeev, J. Liu, C. Gan, A. Kareev, J. Freeland, M. Xiao, and J. Chakhalian, Appl. Phys. Lett. 93(6), 061909 (2008).

[11] D. Aspnes and A. Studna, Phys. Rev. Lett. 54, 1956 (1985).

[12] K. Fleischer, R. Verre, O. Mauit, R. G. S. Sofin, L. Farrell, C. Byrne, C. M. Smith, J. F. McGilp, and I. V. Shvets, Phys. Rev. B 89, 195118 (2014).

[13] R. Verre, K. Fleischer, R. Sofin, N. McAlinden, J. McGilp, and I. Shvets, Phys. Rev. B 83 (2011).

[14] J. Brunen and J. Zegenhagen, Surf. Sci. 389, 349 (1997).

[15] B. C. Russell and M. R. Castell, Phys. Rev. B 77(24), 245414 (2008).

[16] Y. Cao, S. Wang, S. Liu, Q. Guo, and J. Guo, J. Chem. Phys. 137, 044701 (2012).

[17] H. Bando, Y. Aiura, Y. Haruyama, T. Shimizu, and Y. Nishihara, J. Vac. Sci. Technol. B 13, 1150 (1995).

[18] J. A. Enterkin, A. K. Subramanian, B. C. Russell, M. R. Castell, K. R. Poeppelmeier, and L. D. Marks, Nat. Mater. 9, 245 (2010).

[19] Z. Wang, F. Yang, Z. Zhang, Y. Tang, J. Feng, K. Wu, Q. Guo, and J. Guo, Phys. Rev. B 83, 155453 (2011).

[20] U. Rossow, L. Mantese, and D. Aspnes, J. Vac. Sci. Technol. B 14(4), 3070-3074 (1996).

[21] D. Papadimitriou and W. Richter, Phys. Rev. B 72(7), 075212 (2005).

[22] K. Hingerl, R. Balderas-Navarro, A. Bonanni, P. Tichopadek, and W. Schmidt, Applied surface science 175, 769-776 (2001).

[23] D. Aspnes, Y. Chang, A. Studna, L. Florez, H. Farrell, and J. Harbison, Phys. Rev. Lett. 64(2), 192 (1990).

[24] K. Van Benthem, C. Elsässer, and R. French, J. Appl. Phys. 90(12), 6156-6164 (2001).

[25] J. Stoch and J. Gablankowska-Kukucz, Surf. Interface Anal. 17(3), 165-167 (1991).

[26] K. A. Stoerzinger, W. T. Hong, G. Azimi, L. Giordano, Y. L. Lee, E. J. Crumlin, M. D. Biegalski, H. Bluhm, K. K. Varanasi, and Y. Shao-Horn, J. Phys. Chem. C 119(32), 18504-18512 (2015).

[27] Y. S. Kim, J. Kim, S. Moon, W. Choi, Y. Chang, J. G. Yoon, J. Yu, J. S. Chung, and T. Noh, Appl. Phys. Lett. 94(20), 202906 (2009).

[28] V.E. Henrich, G. Dresselhaus, and H. Zeiger, Phys. Rev. B 17(12), 4908 (1978).

[29] K. Eom, E. Choi, M. Choi, S. Han, H. Zhou, and J. Lee, The Journal of Physical Chemistry Letters 8(15), 35003505 (2017).

[30] T. Beck, A. Klust, M. Batzill, U. Diebold, C. Di Valentin, and A. Selloni, Phys Rev Lett 93(3), 036104 (2004).

[31] H. Cheng and A. Selloni, Phys. Rev. B 79(9), 092101 (2009).
[32] B. Walls, O. Lübben, K. Palotás, K. Fleischer, K. Walshe, and I. Shvets, Phys. Rev. B 94(16), 165424 (2016). 